\title{
Multi-modal Microscopy of Almost Nothing - Aerogels
}

\author{
Beer G.A. ${ }^{1}$, Olin A. ${ }^{1}$, Humphrey E.C. ${ }^{2}$
}

1 Department of Physics and Astronomy/TRIUMF, University of Victoria, Victoria, Canada

2 Advanced Microscopy Laboratory, University of Victoria, Victoria, Canada

Aerogels, consisting mostly of air caught in a matrix of low-density strands of Silicon Dioxide, were first made by supercritical drying of gels in the 1930s. When viewed on a white background they almost disappear, and on a black background they appear blue due to Rayleigh scattering. There is essentially no boundary scattering of light off the solid surface. While excellent thermal insulators, they have a multitude of other uses. For purposes outlined below, we required macro, micro, and nano 2D and 3D images of aerogel sheets of densities $>27 \mathrm{mg} / \mathrm{cc}$ (20X air at STP) with modified surfaces.

Critical to a proposal to measure the anomalous magnetic moment of the muon, (a "heavy electron" which is produced at accelerator laboratories), our group had need of a solid, windowless muonium production target [1] similar to powder. A solid aerogel layer should efficiently slow positive muons within the layer where they then capture electrons to form neutral muonium atoms which thermalize and diffuse out of the solid surface into vacuum.

A range of densities of aerogel sheets was manufactured in Japan by our collaborators [2] and early experimental measurements at TRIUMF of the muonium yield in vacuum from these solid sheets showed that significantly less muonium escaped from the solid aerogel surface than was observed from silicon dioxide (Cabosil) powders [3]. Simulations of muonium diffusing from aerogels suggested that modification of the aerogel surface at a scale of hundreds of micrometers might improve the yield. Several surface modification techniques were tried and we settled on using a pulsed laser to "drill" 160 micrometer diameter holes into, but not through, the aerogel surface in a grid pattern. Weight measurements suggested that little of the solid material was lost when the holes were drilled.

To characterize the microstructure of the aerogel surfaces, a Hitachi S-4800 SEM was used with varying degrees of success. Problems encountered and overcome resulted from the tenuous nature of the structure of the aerogel samples. First, our Japanese colleagues and then we prepared early samples with several-nanometer-layers of osmium or carbon to reduce charging with the result that the apparent size of the Silicon Dioxide particles was about 20-40 nm, in disagreement with expectations and with our TEM images of 5-6 nm. Imaging uncoated surfaces rectified this error in image size. However charging of thicker SEM samples caused the surface to go in and out of focus as the target "breathed" due to electrostatic repulsion. This, and obvious charging of the target surface, was reduced or eliminated by choosing thinner samples and a very low electron beam energy of between 500 and $700 \mathrm{eV}$ along with an adjusted condenser setting. Bonding of the $\sim 10 \mathrm{mg}$ samples to stubs with colloidal carbon was facilitated using vacuum tweezers and a grounded mat with connecting wrist straps to eliminate electrostatic interactions while handling the aerogel near metal surfaces.

Optical imaging of patterns of $160 \mathrm{um}$ diameter holes in 30 and $50 \mathrm{mg} / \mathrm{cc}$ aerogel sheets 5 to $7 \mathrm{~mm}$ thick (with optical refractive indices of the order of 1.01) show the cylindrical aerogel surface created by the laser interaction. (This laser interaction creates the optical boundary that enables optical imaging). In 2D, it was difficult to determine that they were holes and not pillars pointing out from the surface Fig. 1. Using the Helicon Focus image processing program on a stack of $\sim 40$ optical images taken with an 
Olympus SZX 16 microscope with a DP71 camera, we obtained in-focus images of arrays of the several-mm-deep holes and created a rotating image built from the image stack to see the 3D effect.

To clarify, a calibrated rotating target holder was built, with the possibility to light the sample at a constant angle from above or below, and in-focus images were created at a series of angles from flat to 25 degrees on a black background. These were used to create stereo and rocking movie images. Images in 2D and 3D of a sample sliced along the axis of the holes showed further details of the hole shape.

These optical images were supplemented by auto-fluorescence Confocal images taken with an Olympus BX61 Wi (with Fluoview). Images taken looking straight down the holes showed the surface clearly but, when computer-rotated through angles up to 90 degrees to show hole shape, the resultant image of the deeper region did not agree with images taken with the target placed at 90 degrees Fig. 2.

To obtain comparative micro surface details, a sheet of $30 \mathrm{mg} / \mathrm{cc}$-drilled aerogel was sliced parallel to the axis of the hole and 3D SEM images were taken of the cylindrical hole surface, the un-drilled aerogel between the holes, and the laser-interaction region Fig. 3. Clear differences are seen in the surface appearance and roughness of these three regions.

In conclusion, optical, confocal, HR-TEM and uncoated SEM surface macro, micro and nano images have been obtained of aerogels, mostly in 3D, to study laser-drilled holes in their surfaces. These images can be used both to understand the effect of surface structure on muonium yields into vacuum and to provide quality control of the fabricated aerogel targets.

[1] Beer G. et al, Prog. Theor. Exp. Phys. $091 \mathrm{C} 01$ (2014) 7 pages

[2] Tabata M et al, Nucl. Inst. and Meth. A 668 (2012) 64-70

[3] Janissen A Phys. Rev. A 42 (1990) 161-169

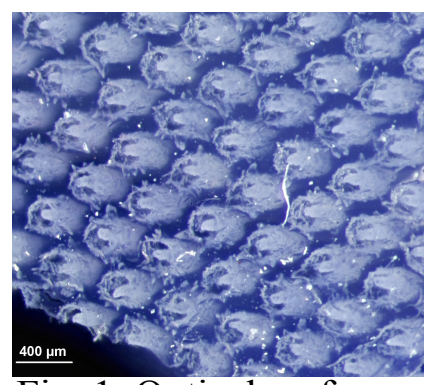

Fig. 1. Optical surface

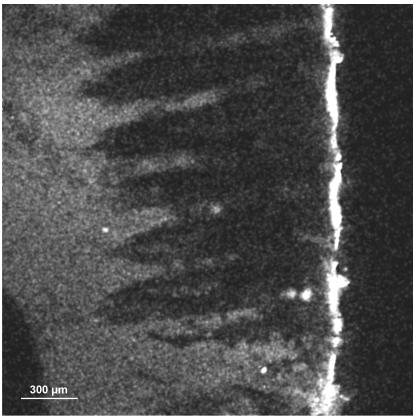

Fig. 2. Confocal slice
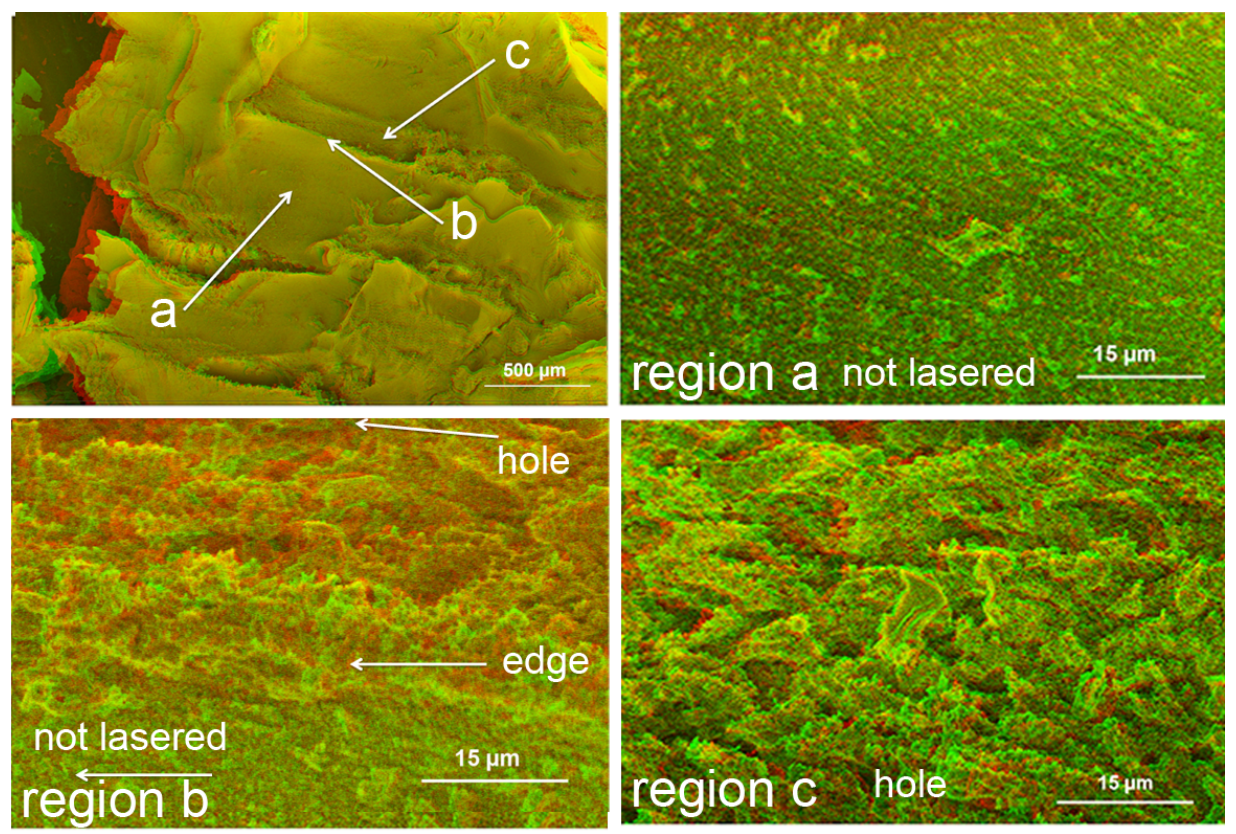

Fig 3. SEM 3D images of hole surface and surrounding aerogel 